\title{
A Cascading Neural Network Ensemble for Locating Faults of Large-scale Information Systems
}

\author{
Chen Zhifeng ${ }^{1}$, Peng Minjing ${ }^{2}$ and $\mathrm{Li} \mathrm{Bo}^{3}$ \\ ${ }^{1}$ Business Support Center of Jiangmen Branch, \\ China Mobile Group Guangdong Ltd., Co. \\ ${ }^{2,3}$ Institute of E-commerce \& Public Information Services, Wuyi University \\ 1491113317@qq.com, ${ }^{2} 15819748999 @ 139 . c o m,{ }^{3}$ li.b@joybirds.cn
}

\begin{abstract}
Failure types of Information Systems are varied, which leads to the long duration for locating faults, and decreases the quality of customer service or income. In order to accurately and timely locating BOSS faults, a cascading neural network ensemble was proposed. In the proposed ensemble, the approach of ensemble was used to promote the accuracy of locating faults, and the cascading structure was employed to decrease the time duration of locating. The proposed approach used in fault location BOSS achieved the following results: (1) the speed of locating faults has been improved; (2) complaint rate from customers has been reduced; (3) the billing losses have been decreased.
\end{abstract}

Keywords: Fault locating, Neural network, Cascading structure, Ensemble, Customer service

\section{Introduction}

Business \& Operation Support Systems (BOSS) are referred to as operational support systems that are the typical large-scale information systems [1]. They are telecom operators' integrated, customer-centric and resource sharing supporting systems. They take customer service, business operation and management as the core, and have the main functions of key business operations: the billing and settlement system, the accounting system, the customer service system and the decision support system etc., they are complex enterprise information systems and Internet service support systems. At any time there is a large amount of data produced in different parts of the systems, therefore, the systems inevitably occur various types of failures. The typical fault types include: multiple billing, leakage billing, users call failure, Internet access failure, and illegal transaction implementation [2]. These failures will have great influence on the telecommunication operator's normal operating income and brand.

Currently, the process dealing with the faults is: after the customer service staff of the telecommunication operator received the fault information the customers submitted, the fault processing task would be handled to the maintenance personnel. The maintenance personnel would identify the fault according to information provided by the customer and the customer service staff to locate the faults, and solve the problem. This is a passive, delaying and oneway problem solving mode. The passive caused by the fact that the operator can't find the faults before the customers' complaint.

At present, the fault processing method is: the fault information telecommunication operator's customer service staff received its customers to submit, the fault processing task to the maintenance personnel, maintenance personnel to confirm the fault according to the customer and the customer service staff to provide information, locate the fault, then solve the 
problem. This is a passive, delaying and one-way mode. Passiveness shows in the fact that the operator must be provided with relevant error information from customer complaints or front desk to find fault. Delay is reflected in that it always happens that only after faults affect users' call or online behaviors, are perceived by the users, and are reported to customer services, then the faults or problems can be processed. And one way shows in the fact that only when the faults damage the benefits of customers, they can be found, when the faults damage the benefits of the operator, they can't be found. Therefore, we need to find an active, timely and bidirectional fault locating method, in order to protect the interests of both the customers and the telecom operator.

Neural network is a kind of commonly used intelligent classification tool [3]. It can be used to identify the object type through the features of the object. In this research, the neural networks are used as the fault recognition tools; suitable business data are used as identification features; and in order to shorten the time duration of fault identification and achieve the purpose of rapid fault locating, the cascading structure to ensemble the neural networks [4].

\section{Related Works}

Fault locating is a kind of classification problem [5]. For the general classification problems, researchers often use the structure of the neural network shown in Figure $1[6]$.

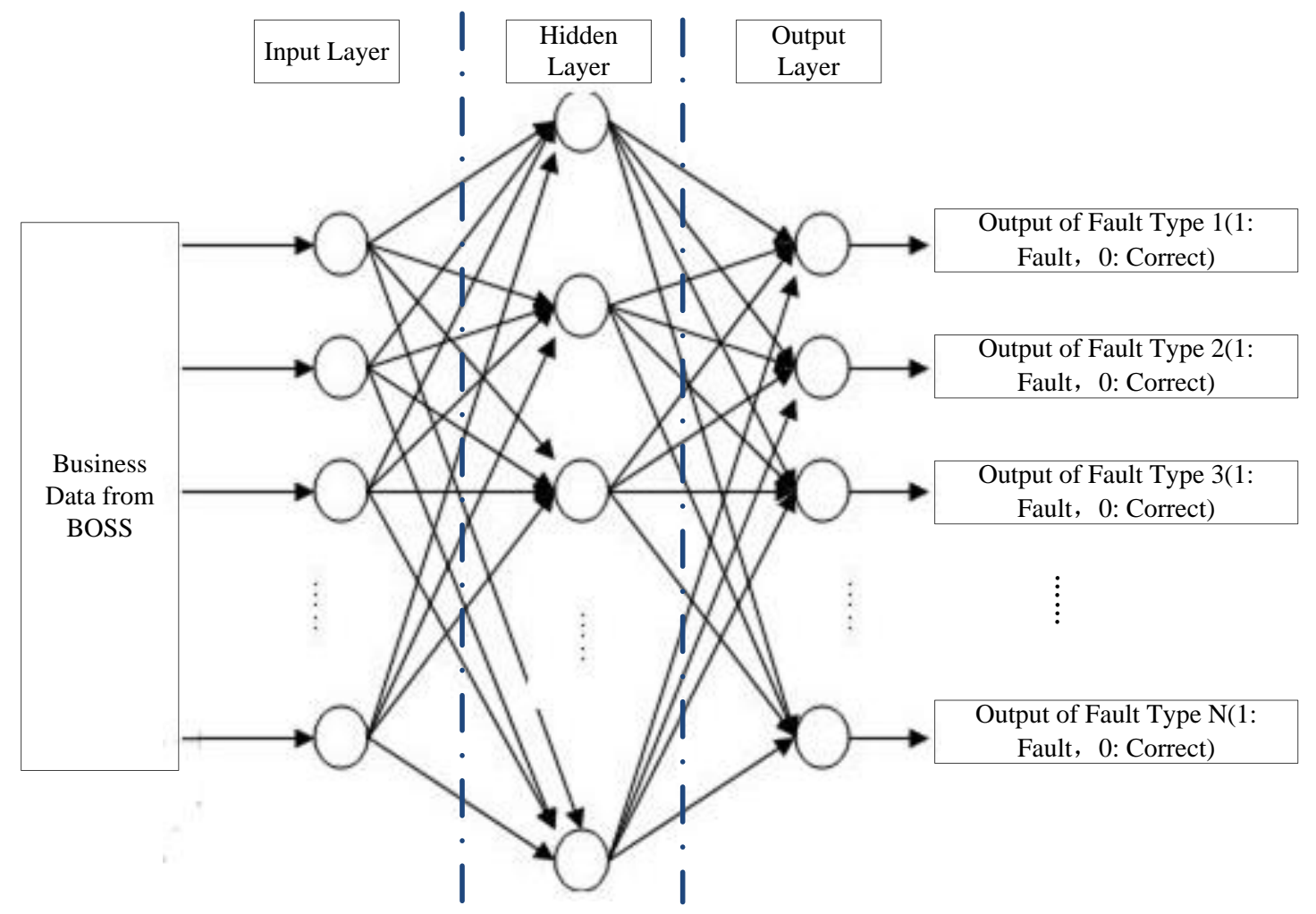

Figure 1. The Commonly used Fault Locating Model based on the Neural Network 
The fault locating model based on neural network in Figure 1 has the following problems:

(1) All types of faults are detected in the same neural network, resulting in the large training sample data are used, which hinders the application practicability of this model. For the input access of the neural network, all the operation data are used for fault locating, and the input signal is representative of all kinds of fault or correct operations of the large information systems. During the training process, any trained feature can't be obtained without all the input training data. Therefore, from the point of view of input and output, a great amount of sample data is required to determine the type of operating fault in the system. This makes the training of the neural network for fault locating need a large amount of sample data, which largely increases the running costs of the large scale information system.

(2) The trained neural network has complex structure to cope with various types of output faults, resulting in largely increased amount of calculation, which seriously delays the fault detection. The purpose of the work of fault locating is to shorten the response time, so the real-time of fault locating is very important. If the structure of the neural network is complex, the computation complexity of training and locating would be increased, and the computing time would be extended, it would extend the time duration of fault locating.

(3) There are no definite established relations between the fault phenomena (neural network output) and the fault causes (neural network input) in the fault locating neural network, which results in that it isn't easy to locating the faults. Because that the BOSS business data of all kinds of different input are in the same input port, and when the output signal produced, we couldn't confirm the relationship between input and output. Therefore, when there are many underlying causes of the faults, it's not easy to locating the fault, which results in that the locating work is not explanatory [7].

\section{General Idea}

The faults are rapidly located in two phases: the training phase and the fault locating phase.

\subsection{The Training Phase}

In the training phase, the following steps are implemented: (1) select the $\boldsymbol{N}$ faults that are critical to the system; (2) conduct correlation tests between the selected output values and the inputs, which stand for the faults. And the input indicators, which represent the fault causes, are selected according to the values of correlation coefficients. The selected input indicators are $\boldsymbol{F}_{n} ;(3)$ for each type of fault, the output data $\boldsymbol{O}_{n}$ and the corresponding input $\boldsymbol{F}_{n}$ are selected to train a single neural network based on the training data samples $\boldsymbol{T} ;$; 4 ) as the neural network ensemble has better classification performance than the single neural network, $\boldsymbol{K}_{n}$ neural networks are selected from the trained neural networks as the units that are integrated into the neural network ensemble $\boldsymbol{E}_{i}$. The output value of $\boldsymbol{E}_{i}$ is used to locate faults [8].

\subsection{The Fault Locating Phase}

The following steps are involved: (1) the neural network ensembles are ranked according to the fault frequencies and the real-time requirements of locating the fault; 
(2) according to the ranking arranged in step (1), the transaction data are used as the input of neural network ensemble $\boldsymbol{E}_{i}$ corresponding to the fault; (3) When the fault couldn't be detected by the ensemble $\boldsymbol{E}_{i}$, the data would be passed on to the next neural network ensemble; if the fault were detected, the cause would be located, and the goal would be achieved.

\section{Algorithm}

The algorithm of the neural network ensemble consists of the following critical steps: (1) Correlation analysis between sample data of fault source and fault phenomenon is conducted. And the input data varies as the fault phenomena varies; (2) the neural network ensembles are trained for locating the single faults; (3) the neural network ensemble is architecture in the cascading form for locating multiple faults.

\section{1) The Input Indicators Selection}

There are many dimensions of input indicators for locating the faults. In order to reduce the computation of neural network ensembles, the reduction of dimension is implemented, which means only suitable feature values that stand for the transaction data of the information system would be selected.

As the optimization strategy, equation (1) is used to conduct the correlation analysis to select the feature values [9]:

$$
r_{X Y}=\frac{\sum_{i=1}^{N}\left(X_{i}-\bar{X}\right)\left(Y_{i}-\bar{Y}\right)}{\sqrt{\sum_{i=1}^{N}\left(X_{i}-\bar{X}\right)^{2}} \sqrt{\sum_{i=1}^{N}\left(Y_{i}-\bar{Y}\right)^{2}}}
$$

where, $\boldsymbol{X}$ are the candidate feature values, $\boldsymbol{Y}$ are the output values related with faults of the system. For example, $X$ can be the ratio for the remaining value of the space with the standard one of the space of hard disk of the server; and Y can be the number of queries per second; Subscript $\boldsymbol{i}$ represents for different time; $\boldsymbol{N}$ represents the number of samples; $\overline{\boldsymbol{X}}$ is the average value of $\boldsymbol{X}_{i} ; \overline{\boldsymbol{Y}}$ is the average value of $\boldsymbol{Y}_{i}$; $\boldsymbol{r}_{X Y}$ represents the correlation between $\boldsymbol{X}$ and $\boldsymbol{Y}$.

In this step, the minimum value of $r_{X Y}$ is set as 0.5 , which means only when the correlation value of the input indicator $\boldsymbol{X}_{i}$ with $\boldsymbol{Y}_{j}$ is larger than 0.5 , the indicator would be used in the training and fault locating phases.

\section{2) The Fault Locater based on the Neural Network Ensemble}

The BP neural network is used to locate the faults. In this algorithm, the network consists of three layers: the input layer, the hidden layer, and the output layer [10]. First, the previously obtained business data or network data are input into the network. After the computation of the hidden layer, the output value can obtain at the output. There are only two possible values: 1 and 0 , where 1 means there is a fault, and 0 means the kind of fault doesn't exist. Based on neural network ensemble theory [11], the approach that neural networks are individually trained and the output values of the networks are integrated into the value of the ensemble, can significantly improve the generalization ability of neural networks. In this research, the generalization ability is the ability of locating the faults. The algorithm of result integration is the AdaBoost. There are several weak classifiers are trained by different data related with a fault. And a strong classifier is achieved by linearly integrating the outputs of these classifiers. In this 
algorithm, each weak classifier is assigned a weight, if a sample is not correctly classified by the classifier; its weight would be increased [12]. If the opposite happened, the weight would be reduced. The details of the algorithm are described as follows:

(1) The positive and negative samples of business data are used as the sample data for training the classifier. Given a certain amount of sample, as sample data to train the classifier. It's assumed that there are $N$ positive samples and negative samples;

(2) In order to initialize the weights of neural networks combined into the ensemble classifier, the ratios with positive samples to negative samples are 9:1, 8:2, 7:3, .., and 5:5. And the combination samples are separately used to train the classifier. The numbers of each group of training data with positive and negative samples are the same;

(3) The single neural networks are separately trained for 100 times;

(4) The simulation errors are estimated for the trained neural networks. The values of the errors are the differences between the training simulation outputs and the actual outputs;

(5) The top three neural networks with the least errors are selected as the networks combined into the neural network ensemble as the classifier;

(6) As shown in Figure 2, the selected individual neural networks are linearly combined into the classifier for locating the faults.

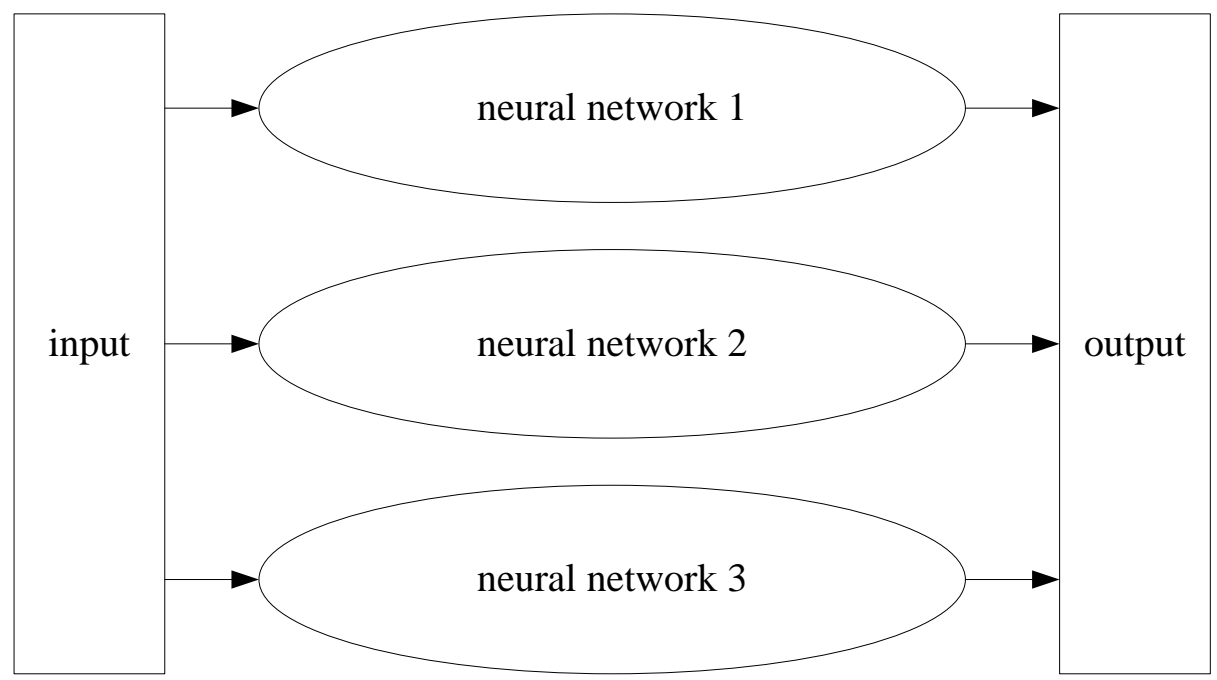

Figure 2. The Neural Network Ensemble

The fault locater could be obtained through the above steps. And the fault locater is able to recognize whether the input data represent the system error and locates the fault. In this way, it improves the efficiency of fault locating. But the classifier can only locate a single fault from the data of the information system.

\section{3) The Classifier for Multiple Faults}

The classifier proposed in the part of 2) is aimed at locating the single fault in the information system. When there are two or more faults in the system, the classifier can't locate them. Therefore, a cascading structure is proposed to architect the ensemble classifier as shown in Figure 3. 
The cascading classifier combines multiple neural network ensembles in series way. Because that the purpose of the proposed classifier is to improve the efficiency of fault recognition and shorten the duration of locating time, the places are arranged according to the frequencies of the corresponding faults: the higher is the frequency, the higher is the priority of locating. In the process of training, the data of both positive and negative samples are used for the corresponding to-be-integrate classifiers.

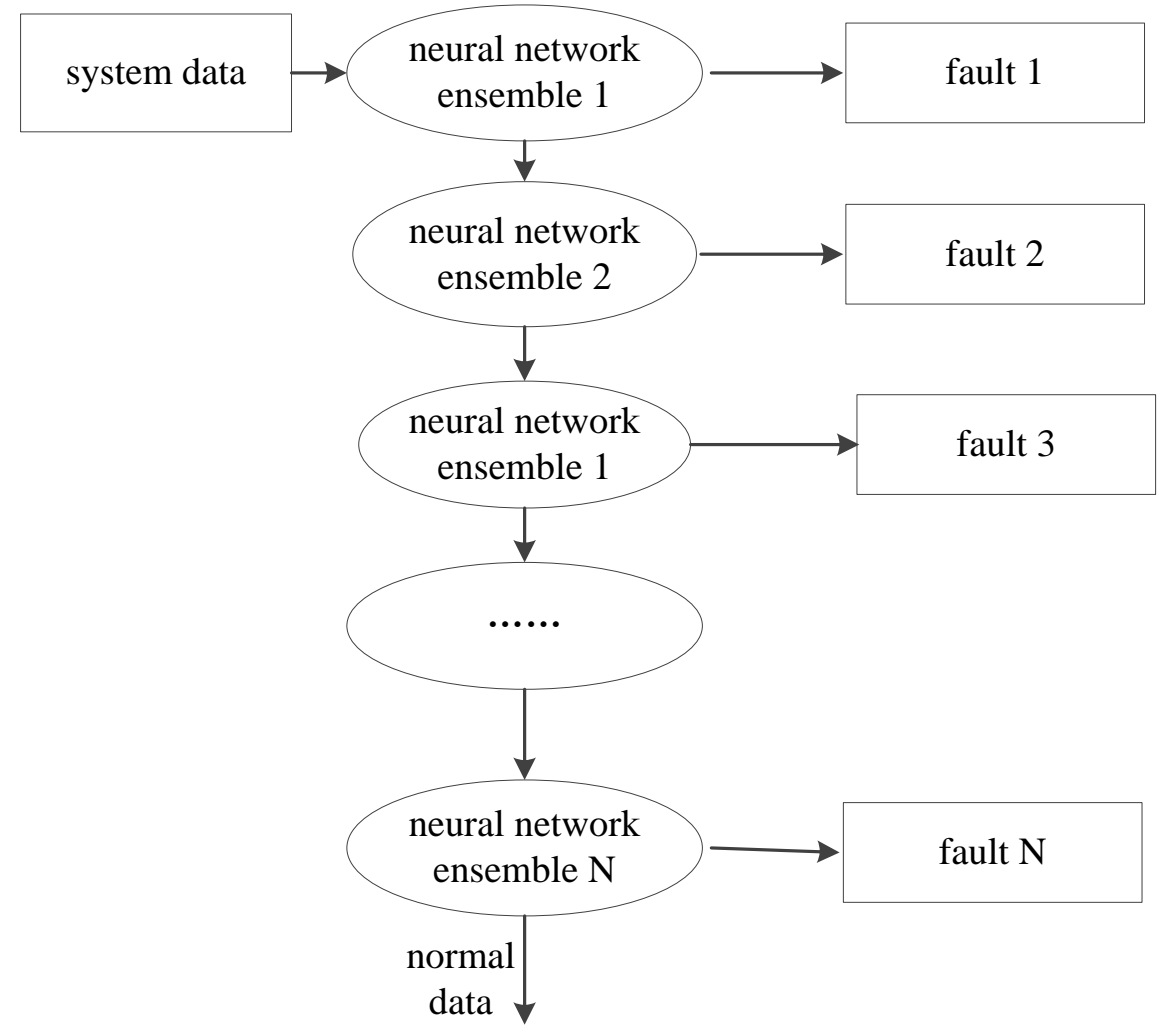

Figure 3. The Cascading Structure of the Integrated Ensemble

The detailed steps are:

(1) The correlation analysis in the process of part 1) is used to select the input indicators. In the analysis, the criteria of selecting the indicators is that their correlation coefficients with the fault $\boldsymbol{Y}$ are greater than 0.5 ;

(2) The fault locating neural network ensembles corresponding to the faults are put into the cascading architecture in Figure 3 according to the frequencies of faults;

(3) The neural network ensembles are separately trained by the sample data from the information system. The process was described in the Steps (1), (2) and (3) of part 2).

Through this process, the information that doesn't contain the fault information would be filtered by the first classifier into the next classifiers in the cascading structure. In this way, the ensemble classifier can efficiently locate the faults and output the causes.

\section{Experiment and Application}

This part consists of two sections: an experiment and an application to the billing system of BOSS. 


\subsection{An Experiment}

In this experiment, the single neural network, the neural network ensemble, and the cascading structured neural network ensemble are separately tested for the average durations of locating the faults.

In the training phase, the number of sample data is 5000. In the sample data, the frequencies with the top fault frequency are 55, 31, 15, 11 and 8 .

After training, the three separately trained fault locators are used to test the 20000 sample data from the same data source. The average duration times are shown in table 1.

Table 1. The Average Duration of Time for Locating Faults

\begin{tabular}{lccc}
\hline fault types & $\begin{array}{l}\text { the single neural } \\
\text { network }(\mathrm{ms})\end{array}$ & $\begin{array}{c}\text { the neural network } \\
\text { ensemble }(\mathrm{ms})\end{array}$ & $\begin{array}{c}\text { the cascading structured } \\
\text { neural network ensemble }(\mathrm{ms})\end{array}$ \\
\hline $\begin{array}{l}\text { fault with the } 1^{\text {st }} \text { higher } \\
\text { frequency }\end{array}$ & 1201 & 1232 & 429 \\
$\begin{array}{l}\text { fault with the } 2^{\text {nd }} \text { higher } \\
\text { frequency } \\
\text { fault with the } 3^{\text {rd }} \text { higher }\end{array}$ & 1019 & 1133 & 774 \\
frequency \\
$\begin{array}{l}\text { fault with the } 4^{\text {rd }} \text { higher } \\
\text { frequency } \\
\text { fault with the } 5^{\text {rd }} \text { higher } \\
\text { frequency }\end{array}$ & 967 & 1367 & 986 \\
\hline
\end{tabular}

The results of Table 1 show that the proposed cascading structured neural network ensemble greatly reduce the duration of time for locating faults.

\subsection{An Application}

The proposed cascading structured neural network ensemble is applied in the fault locating of the billing system of BOSS, as shown in Figure 4.

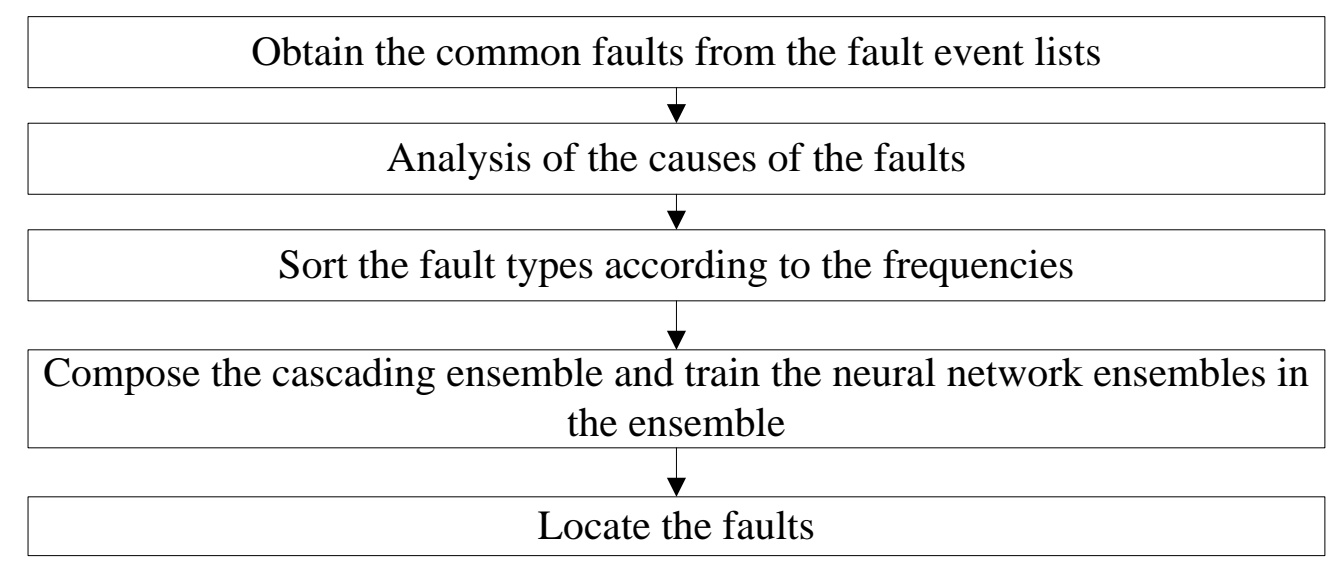

Figure 4. The Application Process

The filtered fault types and corresponding frequencies of the first step in Figure 4 are shown in Table 2. 
Table 2. The Filtered Fault Types and Corresponding Frequencies

\begin{tabular}{|c|c|c|c|c|}
\hline & Filtering items & $\begin{array}{l}\text { The number of } \\
\text { filtered samples }\end{array}$ & $\begin{array}{l}\text { The number of } \\
\text { faults }\end{array}$ & $\begin{array}{l}\text { The fault rate } \\
(\%)\end{array}$ \\
\hline 1 & $\begin{array}{l}\text { The number of users } \\
\text { subscribing and using the } \\
\text { product } E\end{array}$ & 188 & 6 & 3.20 \\
\hline 2 & $\begin{array}{l}\text { The number of payment } \\
\text { records for product } B\end{array}$ & 9714 & 207 & 2.13 \\
\hline 3 & $\begin{array}{l}\text { The number of payment } \\
\text { records for product } A\end{array}$ & 10790 & 128 & 1.19 \\
\hline 4 & $\begin{array}{l}\text { The number of users } \\
\text { subscribing and using the } \\
\text { product } D\end{array}$ & 1188 & 2 & 0.20 \\
\hline 5 & $\begin{array}{l}\text { The number of users } \\
\text { subscribing and using the } \\
\text { product } F\end{array}$ & 1113062 & 1530 & 0.14 \\
\hline 6 & $\begin{array}{l}\text { The number of users } \\
\text { subscribing and using the } \\
\text { product } G\end{array}$ & 20929 & 7 & 0.04 \\
\hline 7 & $\begin{array}{l}\text { The number of users } \\
\text { subscribing and using the } \\
\text { product B }\end{array}$ & 11032 & 1 & 0.01 \\
\hline & Total & 1166903 & 1881 & \\
\hline
\end{tabular}

After the application, the following results were achieved:

(1) The time durations were shortened from the averagely 12 hours to the averagely 4 hours;

(2) Due to the adoption of the fault locating neural network ensemble, the system are capable of locating faults before customers realize the problems;

(3) The application reduced the charging damage. Only for the customized ringing tone, at least 400000 Yuan loss per month has been reduced since March of 2013.

\section{Conclusions}

The BOSS system is a typical large-scale information system. It has various types of faults. The long time duration between the fault's occurrence and location would seriously damage the service quality of telecom operator. In the research, the neural network ensemble is architecture in the cascading structure, which greatly shortens the time durations of locating the faults.

\section{Acknowledgements}

This research work is supported by the Natural Science Foundation of Guangdong Province (10452902001004947) and China Mobile Group.

\section{References}

[1] Z. Xu, X Wang and Q Xie, "Real time control theory of charging and its implementation in BOSS", Telecommunications Science, vol. 3, no. 48, (2003).

[2] X. Fu and M. Song, "Application of IT audit in BOSS revenue assurance", Telecommunications Science, vol. 11, no. 50, (2005).

[3] Z. Zhou, J. Wu and W. Tang, "Ensembling neural networks: Many could be better than all", Artificial intelligence, vol. 1-2, no. 137, (2002). 
[4] Z. Li, Q. Gao, B. He and W. Xie, "A network architecture with controllable service based on Internet", Computer engineering and design, vol. 8, no. 31, (2010).

[5] P. A. Jansson, "Neural networks: An overview", Analytical chemistry, vol. 6, no. 63, (1991).

[6] J. Ren, "ANN vs. SVM: Which one performs better in classification of MCCs in mammogram imaging", Knowledge-based systems, vol. 1, no. 26, (2012).

[7] Z. Gui, Z. Liu and X Yan, "The explanation mechanism of neural networks", Computer science, vol. 3 , no. $25,(\mathbf{1 9 9 8})$.

[8] H. Lee, E. Kim and W. Pedrycz, "A new selective neural network ensemble with negative correlation", Applied intelligence, vol. 7, no. 37, (2012).

[9] L. Du, J. Gao and K. Chen, "Reliability allocation based on fault correlation analysis", Computer integrated manufacturing system, vol. 9, no. 17, (2011).

[10] J. Xin, Y. Jiang and S. Shu, "Personalized recommendation Integrating user preference model and BP neural network", Computer engineering and applications, vol. 2, no. 49, (2013).

[11] Z. Zhou and S. Chen, "Neural network ensemble", Journal of computer, vol. 1, no. 25, (2002).

[12] I. Mukherjee, C. Rudin and R. E. Schapire, "The rate of convergence of AdaBoost", The journal of machine learning research, vol. 1, no. 14, (2013).

\section{Authors}

Chen Zhifeng, he is the director of the business support center of Jiangmen branch, China Mobile Group. His research interest is information system.

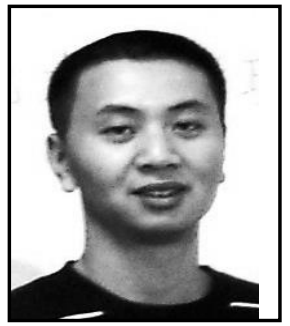

Peng Minjing, he received his M.Sc. in Management (2004) from Jinan University and Ph.D. in Management (2008) from South China University of Technology. Now he is an associate professor of Management at E-commerce Department, Wuyi University. His current research interests include different aspects of Electronic Commerce and Mobile Commerce.

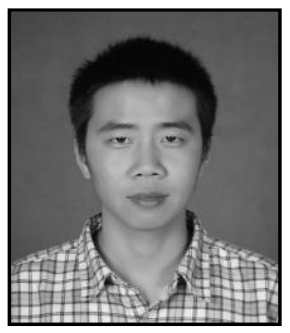

Li Bo, he received his B.Sc. in Management (2011) from Zhengzhou University. Now he is a student working for his M.Sc. in System Engineering at Wuyi University. His current research interests include different aspects of Artificial Intelligence and Electronic Commerce. 
International Journal of Security and Its Applications Vol.8, No.5 (2014) 